\title{
Is vaginal progesterone treatment associated with the development of gestational diabetes? A retrospective case-control study
}

\author{
Klara Rosta ${ }^{1}$. Johannes Ott ${ }^{1}$ (1) $\cdot$ Fanni Kelemen ${ }^{2} \cdot$ Wilhelm Temsch $^{3} \cdot$ Tobias Lahner $^{1} \cdot$ Theresa Reischer $^{1}$. \\ Hanns Helmer ${ }^{1}$. Aniko Somogyi ${ }^{4}$
}

Received: 6 May 2018 / Accepted: 6 September 2018 / Published online: 17 September 2018

(c) The Author(s) 2018

\begin{abstract}
Purpose To determine the incidence of gestational diabetes mellitus (GDM) in pregnant women who received vaginal progesterone due to short cervical length or to prevent recurrent preterm birth.

Methods In this retrospective study, we included 190 women with singleton pregnancies at risk for preterm birth who received vaginal natural progesterone ( $200 \mathrm{mg}$ daily between gestational weeks $16+0$ and $36+0$ ) for a minimum of 4 weeks and delivered $>28$ weeks. The control group consisted of 242 age- and body mass index (BMI)-matched patients without progesterone administration. Data were acquired from a database containing prospectively collected information. Patients with pre-existing diabetes, and conception after in vitro fertilisation procedure were excluded.

Results The incidence of GDM did not differ significantly between the progesterone-treated and the control group (14.7\% vs. $16.9 \%$, respectively; $p=0.597$ ). In a binary regression model, patients with higher pre-pregnancy BMI (OR $1.1 ; p=0.006)$, and those with a family history of diabetes had a higher risk for GDM development (OR $1.8 ; p=0.040$ ), whereas vaginal progesterone treatment had no significant influence $(p=0.580)$.

Conclusion The use of vaginal progesterone for the prevention of recurrent preterm delivery and in women with a short cervix does not seem to be associated with an increased risk of GDM.
\end{abstract}

Keywords Pregnancy $\cdot$ Gestational diabetes mellitus $\cdot$ Preterm birth $\cdot$ Prenatal care $\cdot$ High-risk pregnancy

\section{Introduction}

Gestational diabetes mellitus (GDM), defined as glucose intolerance, first recognized during pregnancy is one of the most common complications during pregnancy. GDM is of high clinical relevance with possible severe adverse outcomes for both mother and child during pregnancy, at childbirth and later in life, which includes increased risks

Johannes Ott

johannes.ott@meduniwien.ac.at

1 Department of Obstetrics and Gynecology, Medical University of Vienna, Waehringer Guertel 18-20, 1090 Vienna, Austria

2 University of Szeged, 12 Dóm tér, 6720 Szeged, Hungary

3 Center for Medical Statistic and Informatics and Intelligent Systems, Medical University of Vienna, Waehringer Guertel 18-20, 1090 Vienna, Austria

4 2nd Department of Internal Medicine, Semmelweis University, Szentkirályi u.46, Budapest, Hungary of preeclampsia, perinatal morbidity, type 2 diabetes and cardiovascular disease [1]. Estimates of GDM indicate that it is a common disease with a reported prevalence of 2-6\% in Europe, although rates may be even higher (e.g. $>10 \%$ in Austria), depending on the selected population and diagnostic criteria applied [2]. Moreover, increasing trends in the prevalence of GDM have been reported in several Western countries, mainly attributed to delayed maternal age at pregnancy as well as to the obesity epidemic. This seems reasonable since advanced maternal age at pregnancy, prepregnancy overweight or obesity belongs to the most important risk factors for GDM [3].

Progestogens (including synthetic progesterone analogues and natural progesterones) are used worldwide to prevent preterm birth, a leading cause of perinatal mortality and morbidity. It has been demonstrated that progesterone is efficient in terms of prolonging pregnancy and improving neonatal morbidity and mortality [4-6]. The use of either synthetic or natural progestogens as a measure to prevent preterm birth has increased substantially in the last several 
years [7]. However, it has been suggested that progesterone might exert diabetogenic effects [8].

Although the diabetogenic effects of progesterone supplementation have been investigated in in vitro $[9,10]$ as well as in retrospective $[8,11,12]$ and prospective [13-15] studies, the results remain inconclusive. Women with an increased risk for preterm delivery due to shortened cervical length with a transvaginal ultrasound scan carried out between $16+0$ and $24+0$ weeks of pregnancy showing a cervical length of $<25 \mathrm{~mm}$ and/or history of a previous preterm birth in our department routinely receive vaginal progesterone treatment starting at 16-20 weeks of gestation until 36 weeks of gestation in an attempt to prolong pregnancy. This allowed us to study the effect of vaginally applied natural progesterone on the incidence of gestational diabetes mellitus which, to the best of our knowledge, has not been studied before. Accordingly, in this retrospective case-control study, we aimed to compare the incidence of GDM between women who had been treated with vaginal progesterone with a matched control group consisting of untreated women.

\section{Materials and methods}

\section{Patient population and study design}

For this retrospective study, we selected women from a databank created within the framework of a screening program for pregnant women at perceived risk for preterm delivery at the Department of Obstetrics and Gynecology of the Medical University of Vienna, a tertiary centre for fetomaternal medicine in eastern Austria [16, 17]. Patients at risk of preterm birth had either a history of one or more spontaneous preterm deliveries or mid-trimester pregnancy losses between $16+0$ and $34+0$ weeks of pregnancy and/or shortened cervical length of $<25 \mathrm{~mm}$ in a transvaginal ultrasound scan performed between $16+0$ and $24+0$ weeks of pregnancy. 387 women were identified between January 2011 to July 2017 , who received vaginal progesterone $(200 \mathrm{mg} /$ day) for a minimum of 4 weeks from the second trimester of their pregnancy. Three hundred age- and BMI-matched pregnant women without progesterone treatment were selected as the control group. For the case and the control groups the following exclusion criteria were applied: pre-existent diabetes mellitus; multiple pregnancies; women with ongoing pregnancies; women with deliveries $<28+0$ weeks; women with a pre-pregnancy BMI $>37$, since the extremely high pre-pregnancy BMI poses a known risk for GDM [18] and often appear together with polycystic ovary syndrome [19, 20]. Moreover, since no data about early oral glucose tolerance tests (OGTTs) were available, we aimed to exclude the majority of early GDM cases. According to the follow-up analysis of the DALI study, the average BMI of women who developed early GDM was $36.2 \mathrm{~kg} / \mathrm{m}^{2}$ [18]. Only three women in the vaginal progesterone treatment group were excluded by applying this criterion, women who had conceived using artificial reproduction treatments, since the latter have been reported to increase the risk of GDM [21-23]. Since progesterone is often provided as high-dose luteal support to women after artificial reproduction treatment, women who were treated for less than 4 weeks with vaginal progesterone, and patients with incomplete data were also excluded from the study. After application of these exclusion criteria, 190 women were finally included in the case group and 242 women in the control group.

\section{Data quality and management}

We used the ViewPoint Fetal Database 5.6.9.17. ${ }^{\circledR}$ software which contains prospectively collected information for data retrieval. For validation of accuracy, we looked at the distribution and ranges of our data. Extreme values were double-checked using source files. In addition, we conducted random checks by two independent investigators to ensure the accuracy of our data. The main outcome measure was the incidence of the diagnosis of GDM. In Austria, GDM is defined as an abnormal $75 \mathrm{mg}$ OGTT performed from weeks $24+0$ to $28+0$ according to the Austrian national guideline and Hyperglycemia and Adverse Pregnancy Outcomes (HAPO) Criteria [24] or elevated blood sugar $(>140 \mathrm{mg} / \mathrm{dl}$ postprandial or $>95 \mathrm{mg} / \mathrm{dl}$ fasting values) at measurements performed later in pregnancy [19]. In addition, the following data were collected: maternal age, pre-pregnancy body mass index (BMI), family history of diabetes mellitus, gravidity and parity, information on whether the women had suffered from GDM in a previous pregnancy, use of betamethasone for lung maturation and tocolysis with atosiban in the evaluated pregnancy, since these medications are considered to influence GDM development [25], gestational age and mode of delivery and birthweight. The study was approved by the Institutional Review Board of the Medical University of Vienna (IRB number: 2191/2017).

\section{Routine care}

Patients with shortened cervical length $<25 \mathrm{~mm}$ in a transvaginal ultrasound scan between $16+0$ and $24+0$ weeks of pregnancy and/or a history of preterm birth underwent regular follow-up examinations for cervical length measurement at our department every 2 weeks until week $34+0$. Patients in the control group had a follow-up visit at gestational week 34 for exact biometry and evaluating the delivery mode and at the onset of labour or at week $40+0$ at the latest. Both the case and the control group underwent regular examinations at a private gynaecologist and obstetrician, in weeks 18-22, 
and 30-34. These follow-up examinations included foetal biometry, evaluation of the placenta, amniotic fluid, dip stick urine test, including glucose, blood tests for virology, blood group, and OGTTs between gestational weeks (gw) 24 and -28 with $75 \mathrm{~g}$ oral glucose load. Concerning late-onset GDM, cases were detected based on clinical signs including glycosuria, polyhydramnion, disproportional growth or foetal abdominal circumference, which are according to guidelines used in German-speaking countries [19]. Patients with these abnormalities were instructed in blood glucose self-monitoring with a four-point blood sugar profile. If the blood sugar profile exceeded the normal values, the patient was diagnosed with GDM. In Austria, pregnant women are required to attend regular appointments in the outpatient care system. Thus, it is likely that any abnormalities between weeks 30 and 34 were recognized and the affected patients referred to their delivery center. Thereby, cases of late GDM development, i.e. after the routine OGTTs, should have become equally evident in the case and the control groups.

\section{Statistical analysis}

Nominal variables are reported as numbers and frequencies, and continuous variables as mean values and standard deviations (SD). Statistical analysis was performed using Pearson's Chi-squared test, Fisher's exact test, and Student's $t$ test, as appropriate on the basis of data distributions to compare differences between the control and the progesterone-treated groups. As pre-pregnancy BMI, family history of diabetes mellitus, tocolysis, and lung maturation with betamethasone are considered to influence the development of gestational diabetes mellitus, multivariate logistic regression was tested to assess relative independent associations on the dependent outcome of GDM incidence. For this analysis, odds ratios (OR), 95\% confidence intervals $(95 \% \mathrm{CI})$ and $p$-values of the likelihood ratio test were calculated. Statistical analysis including matching was performed using the open-source statistical package, IBM SPSS 23. Differences were considered statistically significant if $p<0.05$.

\section{Results}

Detailed characteristics about the included pregnancies are provided in Table 1 . The mean treatment duration with vaginal progesterone was 135 days (range 91-140). Betamethasone for lung maturation as well as tocolysis with atosiban was administered more often in patients with progesterone therapy ( $48.9 \%$ vs. $23.6 \%$, and $48.9 \%$ vs. $17.7 \%$, respectively, both $p \leq 0.001)$. Gestational age at delivery and the rate of preterm delivery before the 37th gestational week differed significantly between the progesterone-treated and control groups $(37.43 \pm 3.88$ vs. $38.66 \pm 3.54$ weeks; $p \leq 0.001$ and delivery before the 37 th week of gestation $34.7 \%$ vs. $25.6 \% ; p=0.039)$. We found no other difference in patient and pregnancy parameters between the two groups.

The incidence of GDM did not significantly differ between women with vaginal progesterone treatment and the control group (progesterone-treated vs. control 28/190, $14.7 \%$ vs. $41 / 24216.9 \%$, respectively; $p=0.597$ ). However, due to the differences in patient and pregnancy characteristics between the case and the control groups, especially concerning the rates of lung maturation and tocolysis, a logistic regression model was used to assess the relative independent predictors for the development of GDM (Table 2). Patients with higher pre-pregnancy BMI (OR $1.1 ; p=0.006$ ), and

Table 1 Basic patient and pregnancy characteristics of the case and control groups

\begin{tabular}{|c|c|c|c|}
\hline & $\begin{array}{l}\text { Women treated with vaginal proges- } \\
\text { terone }(n=190)\end{array}$ & Control group $(n=242)$ & $p$ value \\
\hline Maternal age (years)* & $31.46 \pm 5.91$ & $31.22 \pm 5.51$ & 0.658 \\
\hline Maternal age $>37$ years ${ }^{\#}$ & $30(15.8 \%)$ & $35(14.5 \%)$ & 0.702 \\
\hline Pre-pregnancy BMI $\left(\mathrm{kg} / \mathrm{m}^{2}\right)^{*}$ & $24.0 \pm-4.72$ & $24.16+-4.28$ & 0.718 \\
\hline Previous GDM ${ }^{\#}$ & $24(12.6 \%)$ & $43(17.7 \%)$ & 0.143 \\
\hline Use of atosiban for tocolysis ${ }^{\#}$ & $93(48.9 \%)$ & $43(17.7 \%)$ & $<0.001$ \\
\hline Use of betamethasone for lung maturation ${ }^{\#}$ & $93(48.9 \%)$ & $57(23.6 \%)$ & $<0.001$ \\
\hline Gestational age at delivery (completed weeks)* & $37.43 \pm 3.88$ & $38.66 \pm 3.54$ & $<0.001$ \\
\hline Preterm delivery $<37+0^{\#}$ & $66(34.7 \%)$ & $62(25.6 \%)$ & 0.039 \\
\hline Preterm delivery $<35+0^{\#}$ & $45(23.7 \%)$ & $47(19.4 \%)$ & 0.283 \\
\hline Preterm delivery $<32+0^{\#}$ & $24(12.6 \%)$ & $23(9.5 \%)$ & 0.300 \\
\hline Delivery by caesarean section ${ }^{\#}$ & $79(43.9 \%)$ & $108(44.6 \%)$ & 0.525 \\
\hline Birthweight $(\mathrm{g})^{*}$ & $3004 \pm 767$ & $2996 \pm 869$ & 0.917 \\
\hline
\end{tabular}

Data are presented as *mean \pm standard deviation or ${ }^{\#}$ numbers (percentage)

$B M I$ body mass index, GDM gestational diabetes mellitus 
those with a family history of diabetes had a higher risk for GDM development (OR 1.8; $p=0.040)$. Vaginal progesterone treatment had no significant influence $(p=0.580)$.

In a next step, the study population was divided into BMI subgroups. Again, no differences in the incidence of GDM were found between progesterone-treated and control women: neither in those with a BMI $20.0-24.9 \mathrm{~kg} / \mathrm{m}^{2}(6 / 77$, $7.8 \%$ vs. $15 / 103,14.5 \%$, respectively; $p=0.162$ ), nor in those with a BMI $25.0-29.9 \mathrm{~kg} / \mathrm{m}^{2}(9 / 40,22.5 \%$ vs. $13 / 57,22.8 \%$, respectively; $p=0.972$ ), nor in those with a BMI $\geq 30 \mathrm{~kg} /$ $\mathrm{m}^{2}(7 / 26,26.9 \%$ vs. $20 / 42,47.6 \%$, respectively; $p=0.090)$.

\section{Discussion}

In this retrospective case-control study, the use of vaginal progesterone was not associated with an increased risk of GDM development. This is of clinical importance, since the use of vaginal progesterone for the prevention of preterm birth and for reducing the risk of preterm delivery is widespread and recommended by international guidelines. Notably, the American College of Obstetricians and Gynecologists and SMFM recommended treatment with either 17 OHP-C or vaginal progesterone for women with a prior spontaneous preterm birth to prevent recurrent preterm birth [26]. According to the National Institute for Health and Care Excellence Guidelines, either cervical cerclage or vaginally administered progesterone can be administered in women with a history of previous preterm birth or a shortened cervix [27]. The 2017 SMFM statement reaffirmed that vaginal progesterone should not be considered a substitute for 17 OHP-C in women with a singleton gestation and a history of prior spontaneous preterm birth and proposed the use of vaginal progesterone suppositories $(200 \mathrm{mg}$ ) or progesterone gel $(90 \mathrm{mg})$ in singleton pregnancies with no known preterm birth history and shortened cervix $(<20 \mathrm{~mm})$ [7].

Table 2 Results of the binary logistic regression model for the prediction of GDM development

\begin{tabular}{lrc}
\hline & OR $(95 \%$ CI $)$ & $p$ value \\
\hline Vaginal progesterone treatment & $0.85(95 \%$ CI 0.47-1.52) & 0.580 \\
Pre-pregnancy BMI kg/m ${ }^{2}$ & $1.1(95 \%$ CI 1.02-1.15) & 0.006 \\
Family history of diabetes & $1.8(95 \%$ CI 1.03-3.14) & 0.040 \\
Betamethasone 12 mg 2 $\times$ within & $1.47(95 \%$ CI 0.51-4.22) & 0.471 \\
24 h & & \\
Atosiban* for at least 48 h & $0.79(95 \%$ CI 0.26-2.41) & 0.683 \\
\hline
\end{tabular}

$B M I$ body mass index, OR odds ratio, $95 \%$ CI 95\% confidence interval

*In the atosiban group, women received a bolus injection of $6.75 \mathrm{mg}$ intravenous in $1 \mathrm{~min}$, followed by $18 \mathrm{mg} / \mathrm{h}$ for $3 \mathrm{~h}$, followed by a maintenance dosage of $6 \mathrm{mg} / \mathrm{h}$ for $45 \mathrm{~h}$
However, the high incidence of gestational diabetes in our patient collective (progesterone-treated vs. control 28/190, $14.7 \%$ vs. $41 / 24216.9 \%$, respectively; $p \leq 0.597$ ) mirrors the progressive patient care at the Medical University of Vienna and the accumulation of GDM patients as high-risk pregnancies at our institution. In Austria the HAPO criteria are used for screening of GDM [19]. The incidence of GDM in our study is comparable to the overall frequency of GDM (17.8\%, range 9.3-25.5\%) detected among the 15 centres that participated in the HAPO Study using the new International Association of the Diabetes and Pregnancy Study Groups (IADPSG) criteria [28]. The prevalence of gestational diabetes varies between $1 \%$ and $39 \%$ depending on the specific population, ethnicity and diagnostic criteria used [18, 28-30]. As preterm birth poses a burden on families, primary prevention is quintessential.

Progesterone compounds can be administered orally, intramuscularly and vaginally. Studies on the diabetogenic effect of progesterone supplementation have yielded inconclusive results. A number of studies have suggested that intramuscular administration of 17-alpha hydroxyprogesterone caproate (17 OHP-C) might pose a risk of increasing the incidence of GDM [8,11-13]. On the other hand, a secondary analysis of two randomized placebo-controlled trials of 17 OHP-C found no association with higher rates of GDM in either group [14]. Intramuscularly administered progesterone might affect carbohydrate metabolism in the second trimester of the pregnancy, when progesterone levels are high. Vaginal progesterone might influence carbohydrate metabolism to a lesser extent, since it mainly exerts a local effect on the cervical tissue. Although serum levels of progesterone were not recorded in our study, increase in serum levels after topical application has not been reported [31]. To our knowledge this is the first study to evaluate the possible diabetogenic effect of vaginal progesterone administration on the incidence of GDM.

It could be postulated that the diabetogenic effect of progesterone might be critical in subpopulations with risk factors for GDM. Although our study population was matched for BMI, we divided the study population into BMI subgroups to compare the diabetogenic effect of vaginal progesterone within these subgroups. In contrast to the study of Rebarber et al. [13] we found no difference in the incidence of GDM when comparing the BMI subgroups of progesterone-treated patients and controls. BMI was also included in the binary logistic regression model for the prediction of GDM development and, as expected, patients with higher pre-pregnancy BMI had a higher risk of developing GDM.

Our study is limited by a relatively low sample size. A further limitation of the study is that the case group underwent regular control check-ups every 2 weeks, thus, there might be an observational bias to diagnose GDM after regular OGTT screening based on signs such as glycosuria, 
polyhydramnion, disproportional growth or foetal abdominal circumference [19]. Nonetheless, the Austrian outpatient care system requires a detailed ultrasound examination between gestational weeks 30 and 34 which functions as a checkpoint to recognize any signs of pathology and refer the patient to the delivery center. Our results reflect a good functioning outpatient care system, since the incidence of GDM in the control group did not seem to be underdiagnosed, compared to international standards. Furthermore, this study did not aim to assess the efficacy of vaginal progesterone treatment in the prevention of preterm birth, and we did not aim to fully evaluate foetal outcomes. We were also unable to stratify the data by maternal race or ethnicity, since these data were not routinely recorded in the database.

In conclusion, our data suggest that vaginal progesterone treatment for cervical shortening/risk of preterm birth does not lead to an increased rate of GDM development, at least in women with a BMI $<37.0 \mathrm{~kg} / \mathrm{m}^{2}$ who had conceived naturally. However, further studies are needed to evaluate the diabetogenic effect of widely used natural vaginal progesterone on high-risk populations for GDM.

Acknowledgements Open access funding provided by Medical University of Vienna.

Author contributions All authors substantially contributed to the manuscript: KR conceived the study, participated in study setup, study design, data review and analysis, data management and drafted the manuscript. JO participated in study design and drafted the manuscript. FK performed data review and analysis, data management and drafted the manuscript. TL participated in study design, data abstraction, data review and data analysis and drafted the manuscript. WT participated in data review and data analysis. TR drafted the manuscript. $\mathrm{HH}$ drafted the manuscript. AS participated in study design and drafted the manuscript.

Funding This study was supported by the Hungarian Diabetes Association.

\section{Compliance with ethical standards}

Ethics approval This study was approved by the Institutional Review Board of the Medical University of Vienna (IRB number: 2191/2017) and was valid for one year after approval. The study protocol was in accordance with the Helsinki Declaration and current Austrian law and, thus, neither written nor verbal informed consent was necessary according to the Ethics Committee of the Medical University of Vienna. Therefore, it has not been obtained. The data were de-identified for statistical analysis.

Conflict of interest The authors report no conflict of interest.

Open Access This article is distributed under the terms of the Creative Commons Attribution 4.0 International License (http://creativeco mmons.org/licenses/by/4.0/), which permits unrestricted use, distribution, and reproduction in any medium, provided you give appropriate credit to the original author(s) and the source, provide a link to the Creative Commons license, and indicate if changes were made.

\section{References}

1. Burlina S, Dalfrà MG, Lapolla A (2017) Short- and long-term consequences for offspring exposed to maternal diabetes: a review. J Matern Fetal Neonatal Med 16:1-8

2. Buckley BS, Harreiter J, Damm P, Corcoy R, Chico A, Simmons D et al (2012) DALI Core Investigator Group. Gestational diabetes mellitus in Europe: prevalence, current screening practice and barriers to screening. A review. Diabet Med 29(7):844-854

3. Teh WT, Teede HJ, Paul E, Harrison CL, Wallace EM, Allan C (2011) Risk factors for gestational diabetes mellitus: implications for the application of screening guidelines. Aust N Z J Obstet Gynaecol 51(1):26-30

4. Dodd JM, Jones L, Flenady V, Cincotta R, Crowther CA (2013) Prenatal administration of progesterone for preventing preterm birth in women considered to be at risk of preterm birth. Cochrane Database Syst Rev 31(7):CD004947

5. Meis PJ, Klebanoff M, Thom E, Dombrowski MP, Sibai B, Moawad AH et al (2003) Prevention of recurrent preterm delivery by 17 alpha-hydroxyprogesterone caproate. N Engl J Med. 348(24):2379-2385

6. Norwitz ER, Caughey AB (2011) Progesterone supplementation and the prevention of preterm birth. Rev Obstet Gynecol $4(2): 60-72$

7. Society for Maternal-Fetal Medicine (SMFM) Publications Committee (2017) The choice of progestogen for the prevention of preterm birth in women with singleton pregnancy and prior preterm birth. Am J Obstet Gynecol 216(3):B11-B13

8. Waters TP, Schultz BA, Mercer BM, Catalano PM (2009) Effect of 17alpha-hydroxyprogesterone caproate on glucose intolerance in pregnancy. Obstet Gynecol 114(1):45-49

9. Nunes VA, Portioli-Sanches EP, Rosim MP, Araujo MS, PraxedesGarcia P, Valle MM et al (2014) Progesterone induces apoptosis of insulin-secreting cells: insights into the molecular mechanism. J Endocrinol 221(2):273-284

10. Straub SG, Sharp GW, Meglasson MD, De Souza CJ (2001) Progesterone inhibits insulin secretion by a membrane delimited, nongenomic action. Biosci Rep 21(5):653-666

11. Egerman R, Ramsey R, Istwan N, Rhea D, Stanziano G (2014) Maternal characteristics influencing the development of gestational diabetes in obese women receiving 17-alpha-hydroxyprogesterone caproate. J Obes 2014:563243

12. Kouhkan A, Khamseh ME, Moini A, Pirjani R, Valojerdi AE, Arabipoor A, Hosseini R, Baradaran HR (2018) Predictive factors of gestational diabetes in pregnancies following assisted reproductive technology: a nested case-control study. Arch Gynecol Obstet 298(1):199-206

13. Rebarber A, Istwan NB, Russo-Stieglitz K, Cleary-Goldman J, Rhea DJ, Stanziano GJ et al (2007) Increased incidence of gestational diabetes in women receiving prophylactic 17alphahydroxyprogesterone caproate for prevention of recurrent preterm delivery. Diabetes Care 30(9):2277-2280

14. Gyamfi C, Horton AL, Momirova V, Rouse DJ, Caritis SN, Peaceman AM et al (2009) The effect of 17-alpha hydroxyprogesterone caproate on the risk of gestational diabetes in singleton or twin pregnancies. Am J Obstet Gynecol 201(4):392.e1-5

15. Rouholamin S, Zarean E, Sadeghi L (2015) Evaluation the effect of 17-alpha hydroxyprogesterone caproate on gestational diabetes mellitus in pregnant women at risk for preterm birth. Adv Biomed Res 29(4):242

16. Pils S, Eppel W, Promberger R, Winter MP, Seemann R, Ott J (2016) The predictive value of sequential cervical length screening in singleton pregnancies after cerclage: a retrospective cohort study. BMC Pregnancy Childbirth 16(16):79 
17. Pils S, Eppel W, Seemann R, Natter C, Ott J (2014) Sequential cervical length screening in pregnancies after loop excision of the transformation zone conisation: a retrospective analysis. BJOG 121(4):457-462

18. Egan AM, Vellinga A, Harreiter J, Simmons D, Desoye G, Corcoy $\mathrm{R}$ et al (2017) DALI Core Investigator group. Epidemiology of gestational diabetes mellitus according to IADPSG/WHO 2013 criteria among obese pregnant women in Europe. Diabetologia 60(10):1913-1921

19. Kleinwechter H, Schäfer-Graf U, Bührer C, Hoesli I, Kainer F, Kautzky-Willer A et al (2014) German Diabetes Association; German Association for Gynaecology and Obstetrics. Gestational diabetes mellitus (GDM) diagnosis, therapy and follow-up care: practice Guideline of the German Diabetes Association (DDG) and the German Association for Gynaecology and Obstetrics (DGGG). Exp Clin Endocrinol Diabetes 122(7):395-405

20. Vrbikova J, Hainer V (2009) Obesity and polycystic ovary syndrome. Obes Facts 2(1):26-35

21. Jackson RA, Gibson KA, Wu YW, Croughan MS (2004) Perinatal outcomes in singletons following in vitro fertilization: a metaanalysis. Obstet Gynecol 103(3):551-563

22. Pandey S, Shetty A, Hamilton M, Bhattacharya S, Maheshwari A (2012) Obstetric and perinatal outcomes in singleton pregnancies resulting from IVF/ICSI: a systematic review and meta-analysis. Hum Reprod Update 18(5):485-503

23. Sabban H, Zakhari A, Patenaude V, Tulandi T, Abenhaim HA (2017) Obstetrical and perinatal morbidity and mortality among in vitro fertilization pregnancies: a population-based study. Arch Gynecol Obstet 296(1):107-113

24. Coustan DR, Lowe LP, Metzger BE, Dyer AR, International Association of Diabetes and Pregnancy Study Groups (2010) The
Hyperglycemia and Adverse Pregnancy Outcome (HAPO) study: paving the way for new diagnostic criteria for gestational diabetes mellitus. Am J Obstet Gynecol 202(6):654.e1-6

25. Ostlund I, Hanson U (2003) Occurrence of gestational diabetes mellitus and the value of different screening indicators for the oral glucose tolerance test. Acta Obstet Gynecol Scand 82:103-108

26. Society for Maternal Fetal Medicine Publications Committee (2008) ACOG Committee Opinion number 419 October 2008 (replaces no. 291, November 2003). Use of progesterone to reduce preterm birth. Obstet Gynecol 112(4):963-965

27. Sarri G, Davies M, Gholitabar M, Norman JE (2015) Guideline Development Group. Preterm labour: summary of NICE guidance. BMJ 351:h6283

28. Sacks DA, Hadden DR, Maresh M, Deerochanawong C, Dyer AR, Metzger BE et al (2012) HAPO Study Cooperative Research Group. Frequency of gestational diabetes mellitus at collaborating centers based on IADPSG consensus panel-recommended criteria: the hyperglycemia and adverse pregnancy outcome (HAPO) Study. Diabetes Care 35(3):526-528

29. King H (1998) Epidemiology of glucose intolerance and gestational diabetes in women of childbearing age. Diabetes Care 21(Suppl 2):B9-B13

30. Murgia C, Berria R, Minerba L, Malloci B, Daniele C, Zedda $P$ et al (2006) Gestational diabetes mellitus in Sardinia: results from an early, universal screening procedure. Diabetes Care 29(7):1713-1714

31. O'Leary P, Feddema P, Chan K, Taranto M, Smith M, Evans S (2000) Salivary, but not serum or urinary levels of progesterone are elevated after topical application of progesterone cream to pre-and postmenopausal women. Clin Endocrinol (Oxf) 53(5):615-620 